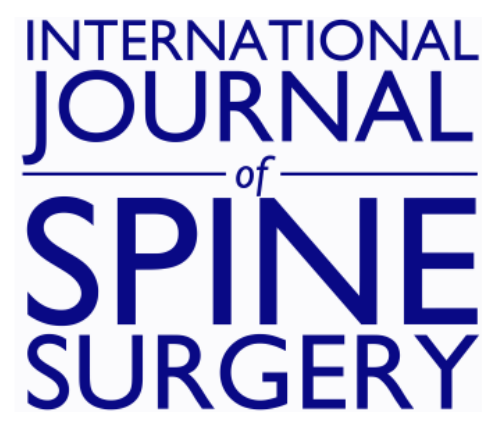

\title{
Full-Endoscopic Oblique Lateral Lumbar Interbody Fusion: A Technical Note With 1-Year Follow-Up
}

Zhen-Zhou Li, Jin-Chang Wang, Zheng Cao, Hong-Liang Zhao, Kai-Uwe Lewandrowski and Anthony Yeung

Int J Spine Surg 2021, 15 (3) 504-513

doi: https://doi.org/10.14444/8072

http://ijssurgery.com/content/15/3/504

This information is current as of April 25, 2023.

Email Alerts Receive free email-alerts when new articles cite this article. Sign up at: http://ijssurgery.com/alerts 


\title{
Full-Endoscopic Oblique Lateral Lumbar Interbody Fusion: A Technical Note With 1-Year Follow-Up
}

\author{
ZHEN-ZHOU LI, MD, ${ }^{1}$ JIN-CHANG WANG, MD, ${ }^{1}$ ZHENG CAO, MD,${ }^{1}$ HONG-LIANG ZHAO, MD, ${ }^{1}$ \\ KAI-UWE LEWANDROWSKI, MD, ${ }^{2}$ ANTHONY YEUNG, MD ${ }^{3}$ \\ ${ }^{I}$ Department of Orthopedic Surgery, the Fourth Medical Center of Chinese PLA General Hospital, Beijing, China ${ }^{2}$ Center for Advanced Spine Care of Southern \\ Arizona and Surgical Institute of Tucson, Arizona; Visiting Professor Department of Orthopaedics, Fundación Universitaria Sanitas, Bogotá, D.C., Colombia; and \\ Department of Neurosurgery, Universidade Federal do Estado do Rio de Janeiro, Rio de Janeiro, Brazil ${ }^{3}$ Desert Institute for Spine Care, Phoenix, Arizona; \\ Executive Director International Intradiscal Therapy Society, Phoenix, Arizona
}

\begin{abstract}
Background: Oblique lateral lumbar interbody fusion (OLLIF) is a minimally invasive lumbar interbody fusion procedure using a bullet-shaped polyetheretherketone (PEEK) nonexpandable fusion cage modified to diminish risk to the exiting nerve root during posterolateral implantation through the Kambin safe zone under fluoroscopic guidance. The objective of this study was to present feasibility of this procedure and 1-year clinical outcome data.

Methods: The authors present a prospective cohort study of 20 patients who underwent fluoroscopy-guided and full-endoscopic OLLIF in 22 segments allowing protection of the exiting nerve root from January 2018 to March 2019. The foraminoplasty, discectomy, endplate preparation, placement of bone graft and insertion of the fusion cage was done under continuous full-endoscopic visualization. The OLLIF fusion was backed up with bilateral percutaneous posterior supplemental pedicle screw fixation. Primary clinical outcome measures were the visual analog scale (VAS) of low back and leg pain, and Oswestry disability index (ODI) at 1 week, 3 months, 6 months, and 1 year after the operation. At final follow-up, the Macnab score was also evaluated. Secondary outcome measures were computed tomography (CT) assessment fusion using the Mannion classification of spinal fusion and adverse events related to the device as well as magnetic resonance imaging (MRI) assessment of nerve root decompression.

Results: All patients had significant relief of low back pain and leg pain, by VAS and ODI scores that improved significantly $(P<.01)$. There were no complications. Postoperative lumbar MRI of all patients showed sufficient direct nerve decompression. At 1-year follow-up, excellent Macnab outcomes were obtained 13 patients, good in six, and fair in one. Impaired sensation and muscle strength of the involved nerve root significantly recovered in all but 2 patients $(P<.05)$. According to the Mannion CT-based classification of spinal fusion, CT showed complete interbody fusion achieved in all 22 segments.
\end{abstract}

Conclusions: Full-endoscopic OLLIF is a safe, effective, minimally invasive, economical, practical, and widely applicable minimally invasive interbody fusion technique in the lumbar spine.

Level of Evidence: 3.

Minimally Invasive Surgery

Keywords: lumbar interbody fusion, full-endoscopic, minimally invasive surgery, lumbar instability, low back pain, lumbar spondylolisthesis, lumbar spinal stenosis

\section{INTRODUCTION}

Oblique lateral lumbar interbody fusion (OLLIF) is a minimally invasive lumbar interbody fusion procedure popularized by Abbasi and Abbasi. ${ }^{1}$ The authors were interested in employing their endoscopic technology platform via the transforaminal approach to aid in the establishment of a minimally invasive surgical corridor to the intervertebral space. Traditionally, OLLIF is performed under the guidance of fluoroscopy by placing instruments and implants through the Kambin triangle. In attempt to reduce radiation exposure to patient and surgeon and to further simplify the procedure, the intervertebral space is evacuated under direct endoscopic visualization. The endplate preparation can be directly inspected and the interbody fusion cage's position can be evaluated visually in addition to standard fluoroscopy images. Moreover, the endoscope can be used to insert the bone graft around the interbody cage.

Most OLLIF cages are bullet-shaped nonexpandable polyetheretherketone (PEEK) implants. During their insertion, traction injury of the exiting nerve root is of some concern, particularly if the surgeon 
only relies on fluoroscopy to determine the position of the instruments and implants and if the patient is under general anesthesia. Neurophysiological monitoring, including somatosensory-evoked potentials, transcranial motor-evoked potentials, and electromyography may be used throughout the operation to aid in safe execution of the operation. However, the threshold for the use of neurophysiological monitoring in the intervertebral foramen region is not standardized. Others prefer local anesthesia as does the corresponding author of this publication. Medical comorbidities in the elderly may be an additional consideration for employing local anesthesia and sedation, which makes the application of endoscopic surgery techniques even more relevant as means to further reduce access trauma and the pain patients may experience postoperatively. Reduction of postoperative dysesthesia due to irritation of the dorsal root ganglion of the exiting nerve root at the surgical level appeared another very reasonable justification for the use of endoscopy during the OLLIF surgery. In early clinical practice, transient postoperative paresthesia was reported to occur in $20 \%-25 \%$ of OLLIF cases, for which reason foraminoplasty was suggested. ${ }^{2}$

In the following, the authors report the primary clinical and secondary radiographic outcome measures at a 1-year follow-up. In this prospective cohort study, the authors aimed to describe the technical aspects of the full-endoscopic OLLIF procedure.

\section{METHODS}

\section{Patients and Indications}

Twenty patients were treated with full endoscopic OLLIF between January 2018 and March 2019 in orthopedic departments of the Fourth Medical Center of Chinese PLA General Hospital for commonly accepted indications for the lumbar interbody fusion of the lumbar spine with or without symptomatic nerve compression.

\section{Inclusion Criteria}

Patients that had the following conditions were included in the study:

(1) chronic low back pain, with or without neurological symptoms in the lower extremity;
(2) failed symptom relief after more than 3 months of nonsurgical treatment;

(3) end-stage degenerative disc (Figure 1A,B) including high-intensity zone on posterior annulus fibrosus, Modic change of endplate (Figure 2A,B);

(4) lumbar segmental instability, with or without spinal stenosis (Figure 2B,C); grade 1- or 2degree lumbar degenerative or isthmic spondylolisthesis (Figure 3A,B); or

(5) symptoms, signs, and physical examination with supportive findings in the preoperative imaging studies.

\section{Exclusion Criteria}

Patients were excluded from the study if they had any of the following conditions:

(1) grade 3 or higher lumbar spondylolisthesis,

(2) skin or deep tissue infections in the surgical area,

(3) unmanageable coagulopathy, or

(4) decompensated cardiopulmonary disease not permitting general anesthesia.

\section{Study Design}

The authors present a prospective cohort study of 20 patients who underwent full-endoscopic and fluoroscopy-guided OLLIF. Institutional Review Board approval was obtained at the Fourth Medical Center of Chinese PLA General Hospital. Patients provided informed consent and were educated about the study design, clinical protocols, and the details of the surgery, including the rationale of treatment, expected outcomes, potential risks, side effects, and the possible need for additional interventional or surgical treatment.

\section{Interbody Fusion}

All operations in this series were performed under general anesthesia with tracheal intubation. Patients were positioned prone on a radiolucent Jackson spinal table. According to the technique described by Yeung et al, ${ }^{3}$ under the guidance of C-arm fluoroscopy, the position of the surgical incision was determined. Percutaneous puncture and foraminoplasty were performed at lower half of foramen with a safe trephine system, according to the method used by $\mathrm{Li}$ et al. $^{4-6}$ The establishment of a safe working channel between skin and 


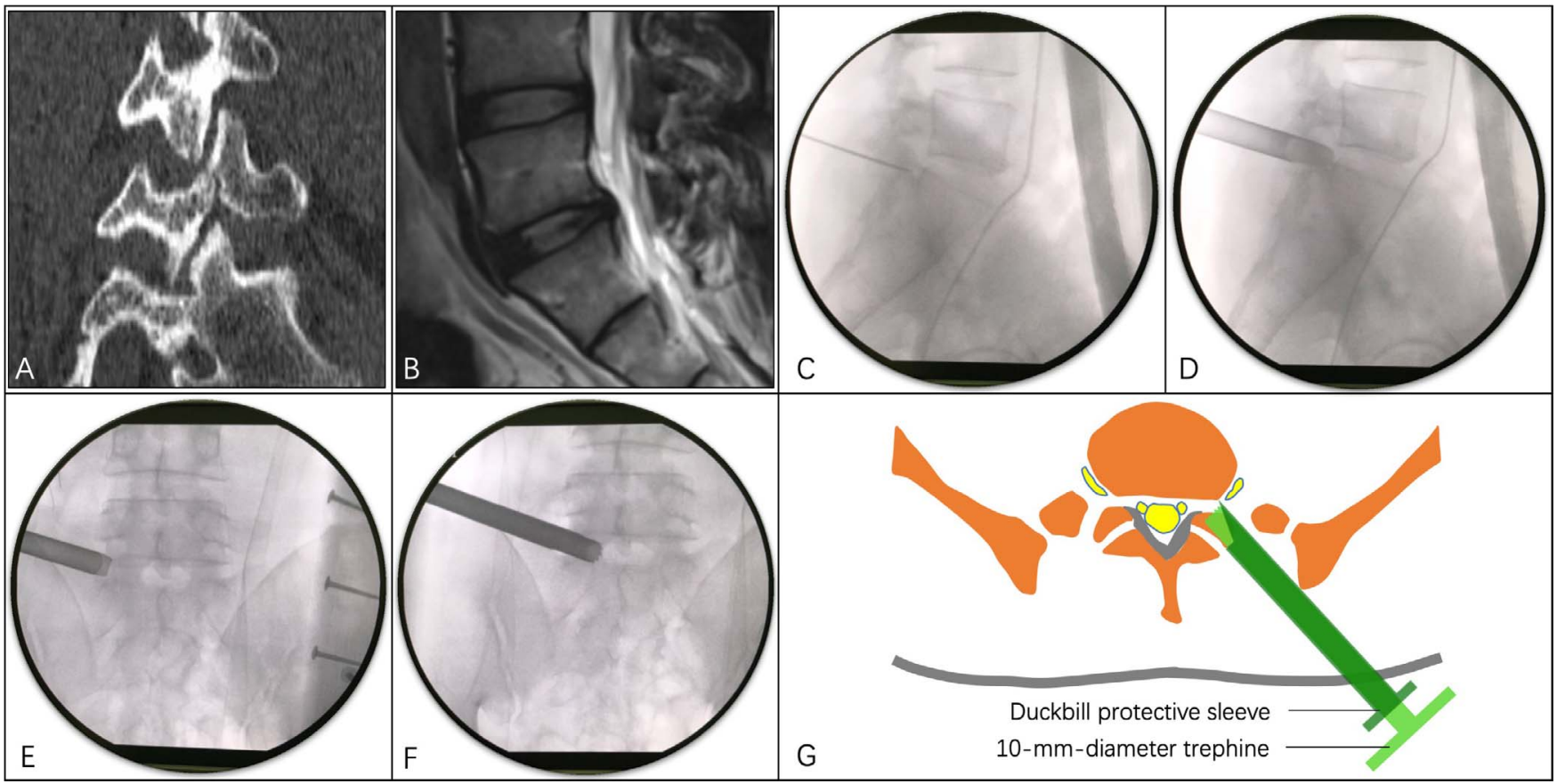

Figure 1. Case 1: a 32-year-old male with discogenic low back pain and spondylolysis. (A) Preoperative computed tomography sagittal reconstruction showed L5 spondylolysis. (B) Preoperative sagittal T2-weighted magnetic resonance imaging showed a degenerative, bulging L5-S1 disc. (C) Percutaneous puncture to the intervertebral foramen under fluoroscopy guidance. The needle tip was located at the posterior upper edge of the lower vertebral body in the lateral view. The direction was parallel to the intervertebral space, located on the central line of the pedicle under the anteroposterior fluoroscopy. A guidewire was introduced along the puncture needle. (D, E) A beveled working sleeve was placed along the soft tissue dilator, and the position of the protective sleeve was confirmed again. (F) A 10-mm-diameter trephine was used in the protective sleeve to remove the lateral and ventral bone from the superior articular process and use it for interbody bone grafting. (G) The schematic diagram showed the working zone of the protective sleeve and trephine. The beveled tip of the protective sleeve protected the exiting nerve root, and the traversing nerve root protected by the ligamentum flavum.
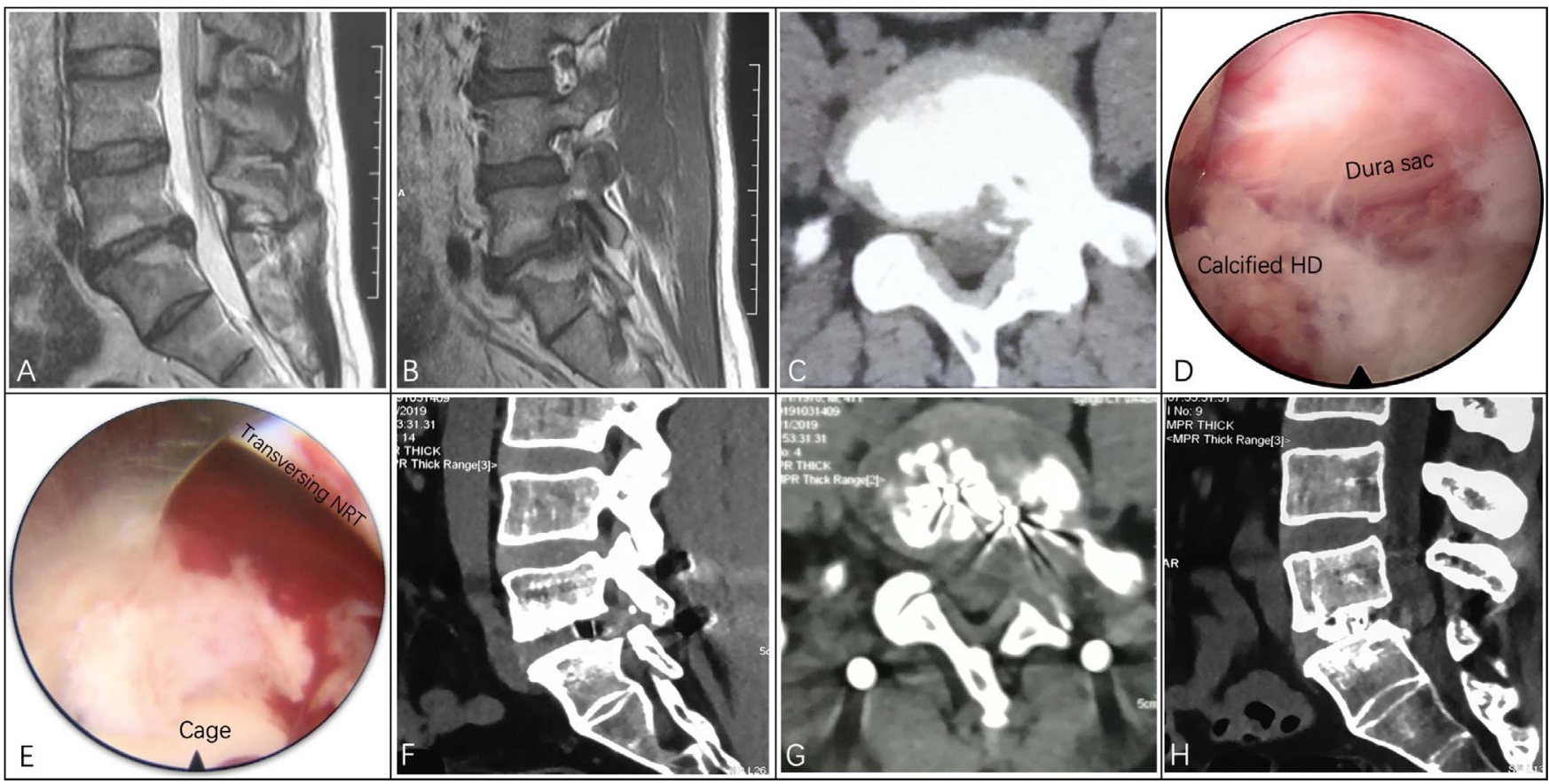

Figure 2. Case 2: a 56-year-old male with L5-S1 Modic changes and lateral recess and foraminal stenosis. (A, B) Preoperative lumbar magnetic resonance imaging showed Modic changes in the upper and lower endplates of the L5-S1 disc and left foraminal stenosis. (C) The preoperative axial computed tomography (CT) scan showed calcified herniated disc compression on the left S1 nerve root. (D) Full-endoscopic exposure of calcified herniated disc. (E) The full-endoscopic view showed the positional relationship between the decompressed nerve root and the fusion cage. (F) Postoperative sagittal CT reconstruction showed left foraminal decompression. (G) Postoperative axial CT showed sufficient decompression of left lateral recess and foramen. (H) Postoperative sagittal CT reconstruction showed L5-S1 interbody fusion. Orientation of the field of view under full endoscope: left, cephalad side; right, caudal side; upper, medial side; lower, lateral side (left side). HD indicates herniated disc; and NRT, nerve root. 

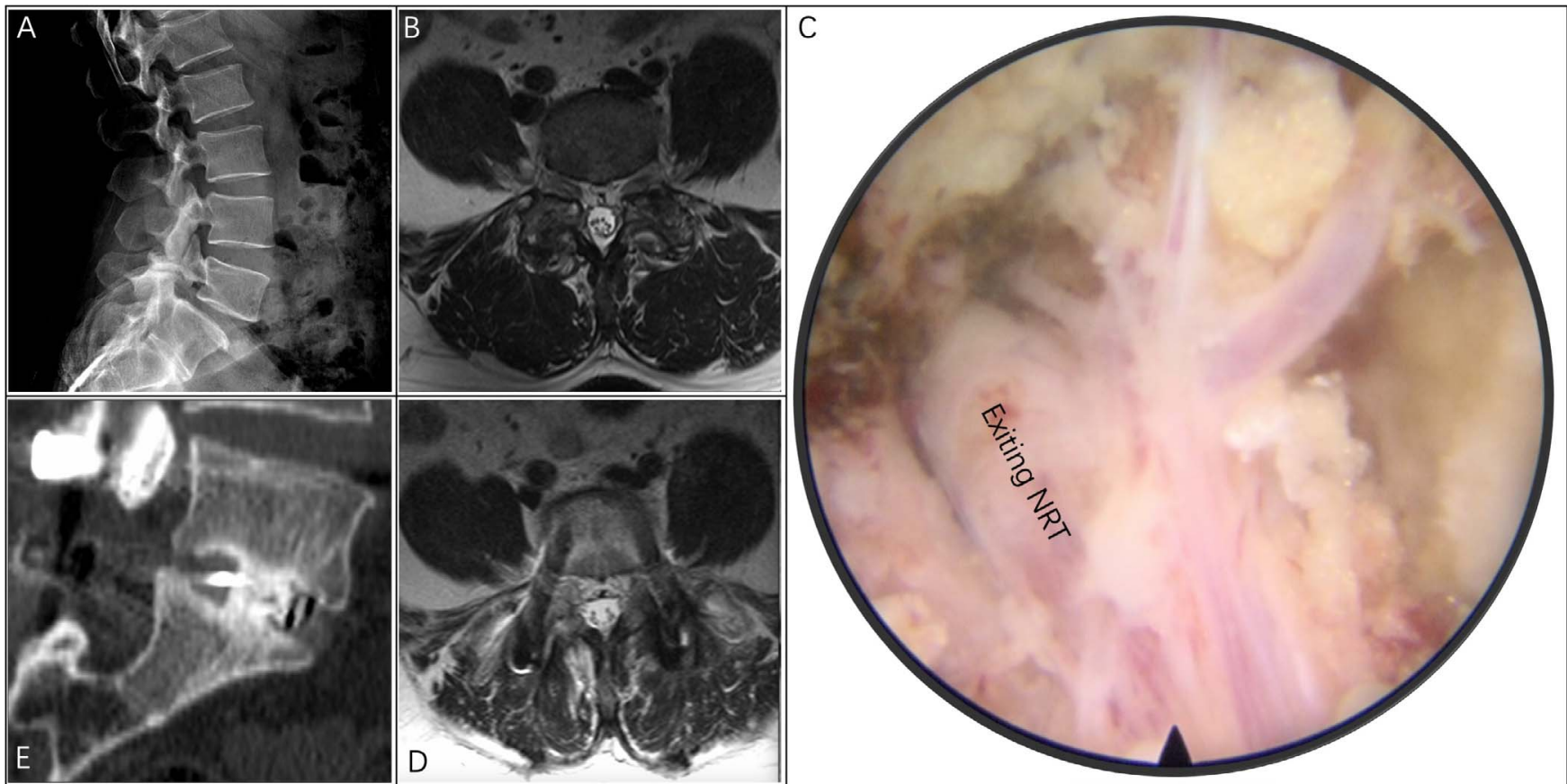

Figure 3. Case 3: a 62-year-old male with isthmic spondylolisthesis. (A) Lateral radiography showed isthmic spondylolisthesis at the L5 level. (B) Preoperative axial T2-weighted magnetic resonance imaging (MRI) showed bilateral foraminal stenosis. (C) Full-endoscopic view of decompressed left L5 nerve root. (D) Three-month postoperative axial T2-weighted MRI showed bilateral foraminal decompression. (E) One-year postoperative sagittal computed tomography reconstruction showed an anatomical reduction of spondylolisthesis and solid interbody fusion. Orientation of the field of view under full endoscope: left, cephalad side; right, caudal side; upper, dorsomedial side; lower, ventrolateral side (left side). NRT indicates nerve root.

disc was established (Figure $1 \mathrm{C}-\mathrm{G}$ ). Throughout the surgery, the exiting nerve root was protected as the annulus fibrosus was exposed, and during the resection of the nucleus pulposus during discectomy. Under continuous full-endoscopic visualization, the endplates were decorticated down to the subchondral (Figure 4A-C). The endoscopic working sheath was used as a bone grafting funnel to place bone graft into the intervertebral space by filling it with pressed allograft cancellous bone material and collagen sponges containing recombinant human bone morphogenetic protein-2 (rhBMP-2). The latter were placed in the most anterior part of the prepared intervertebral disc space close to the anterior longitudinal ligament (Figure 4D). Then, the OLLIF fusion cage (OFuse, Bonovo, Bloomington, Minnesota) filled with rh-BMP-2 and autograft bone was inserted (Figure 4E). Full-endoscopic exploration of the exiting nerve root in the intervertebral foramen was performed (Figure 4F).

\section{Full-Endoscopic Decompression}

The full-endoscopic decompression was done unilaterally to alleviate the traversing nerve root compression and the central spinal canal to its midportion and the ventral or dorsal aspect of the traversing nerve root (Figure 2D-E) ${ }^{5,6}$ For the patients with central stenosis caused by bilaterally hypertrophied flavum ligament and facet joints, fullendoscopic unilateral laminotomy for bilateral decompression was performed through an interlaminar approach. ${ }^{7}$ In patients with isthmic spondylolisthesis, the exiting nerve was exposed via foraminoplasty and decompression of the isthmic pseudarthrosis (Figure 3C). Following satisfactory hemostasis, the full-endoscopic interbody fusion was commenced.

\section{Percutaneous Posterior Supplemental Fixation}

The pedicle screw system (Voyager system, Medtronic, Canifornia) was placed under x-ray fluoroscopy according to standard techniques and fixed with a connecting rod (Figure 5A,B). If no satisfactory reduction of lumbar spondylolisthesis was achieved after implanting the cage, the percutaneous pedicle screw system (Sextant-II system, Medtronic) was further used for additional reduction manipulation (Figure 3E). Final intraoperative $\mathrm{x}$-rays were employed to reconfirm the acceptable implant position. 


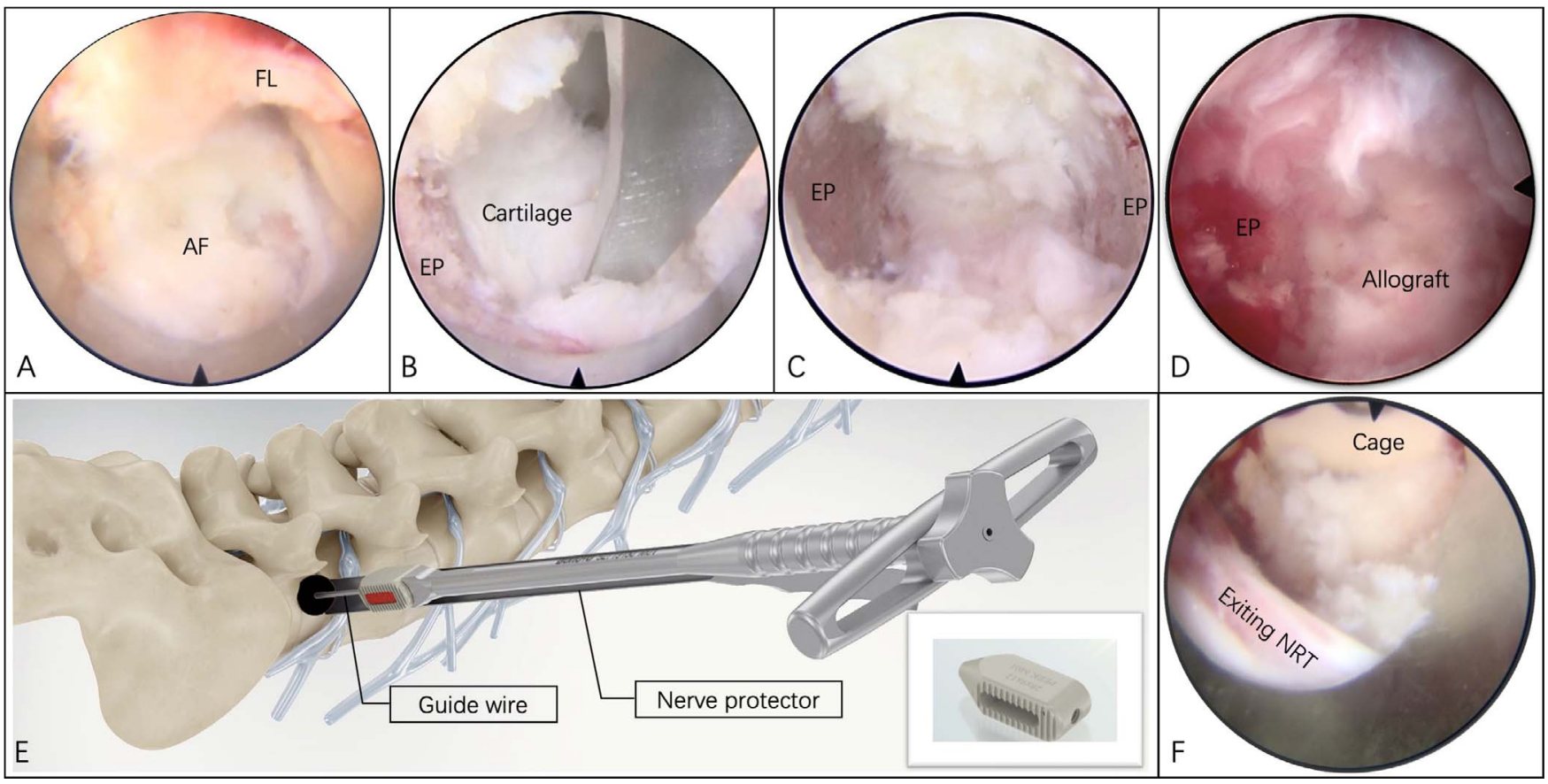

Figure 4. Case 1, continued: surgical technique steps. (A) Rotate the working sheath under full-endoscopic surveillance and protect the exiting nerve root with the tongue end of the working sheath. (B) Use a curette to scrape the cartilage endplate of the upper and lower vertebral bodies under a full endoscope. (C) Prepare the bony endplates to expose the subchondral bone. (D) Place the allograft and rh-BMP-2 into the anterior half of the intervertebral space under press-fit. (E) Insert a guidewire into the intervertebral space the beveled endoscopic working sheath to protect the exiting nerve root by rotating it out of the way. Place a trial for sizing. Insert the bullet-shaped interbody fusion cage filled with autogenous bone and rhBMP-2 over the guidewire. (F) Explore the finished interbody fusion to directly visualize the exiting nerve root and define its relationship with the fusion cage. Orientation of the field of view under full endoscope: left, cephalad side; right, caudal side; upper, medial side; lower, lateral side (left side). FL indicates flavum ligament; AF, annulus fibrosis; EP, endplate; and NRT, nerve root.

\section{Primary Outcome Measures}

Patients' clinical improvements were evaluated at 1 week, 3 months, 6 months, and 1 year after surgery to assess pain symptoms relief. Primary outcome measures were the visual analog scale (VAS, 100point scale) and the Oswestry disability index (ODI). At the final follow-up, Macnab outcomes and the recovery of nerve root function (sensory, muscle strength and tendon reflex) were recorded.

\section{Secondary Outcome Measures}

The lumbar magnetic resonance imaging (MRI) or computed tomography (CT) was reexamined on the second day and 3 months after surgery to evaluate the nerve decompression (Figures $2 \mathrm{~F}-\mathrm{H}$ and 3D). Six months and 1 year after surgery, lumbar CT and 2-dimensional reconstruction were performed to evaluate interbody fusion (Figures $5 \mathrm{C}, \mathrm{D}$ and $3 \mathrm{E}$ ). Fusion was graded based on the method reported by Mannion et al. ${ }^{8}$ Complete fusion was described if trabecular bone was seen to bridge the disc space, with accompanying remodeling of the cortical endplates. Partial fusion was described if trabecular bone could be seen extending from the endplate into the disc space, but the bridge was not complete. Absent fusion was described if the disc space demonstrated no evidence of trabecular bone formation extending from the endplates. If evidence for fusion was considered to be incomplete or absent at the 6-month follow-up, a further CT scan was arranged at 12 months.

\section{Statistical Analysis}

SPSS 26 software (SPSS Inc., Chicago, Illinois) was used for statistical analysis. One-way analysis of variance was performed on VAS scores of low back pain and leg pain, and ODI scores before surgery, 1 week, 3 months, 6 months, and 1 year after surgery. Multiple comparisons at different time points were performed using the least significant difference test. The $\chi^{2}$ test qualitative data of nerve root function status before and 1 year after surgery were compared. A $P$ value of less than .05 was considered statistically significant.

\section{RESULTS}

A total of 20 patients were included in this study, including 17 males and 3 females. The average age 


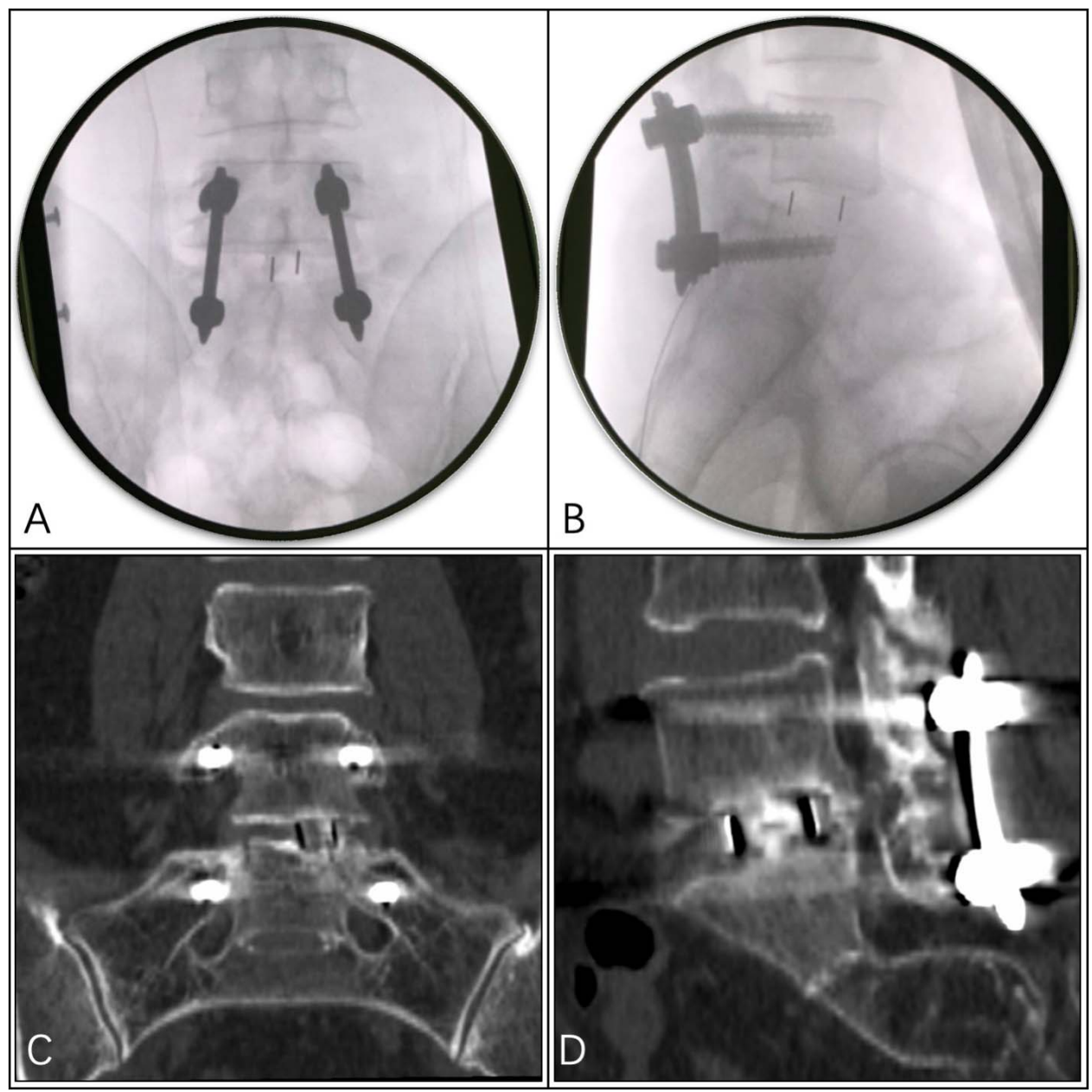

Figure 5. Case 1, continued. (A, B) Anteroposterior and lateral fluoroscopic view showed the position of cage and pedicle system. (C, D) One-year postoperative coronal and sagittal computed tomography reconstruction showed solid interbody fusion.

was 37.2 years, ranging from 20 to 63 years. In terms of surgical indications, there were 2 patients with grade I lumbar degenerative spondylolisthesis with associated central spinal stenosis, and another 2 patients of grade I isthmus spondylolisthesis. Five patients had advanced degenerative disc disease of type II Modic changes. Three patients of these 5 had lumbar disc herniation refractory to conservative care, and another 2 patients suffered from symptomatic unilateral lateral recess stenosis. Six patients underwent the full-endoscopic OLLIF fusion surgery for painful lumbar internal disc disruption, including 2 patients with lumbar spondylolysis and 1 patient with central spinal stenosis. The remaining 5 surgical interventions were done patients suffering from a herniated disc with associated lumbar instability. A total of 22 diseased segments were treated in the 20 study patients with the full endoscopic OLLIF surgery. The surgical levels were L1-2, L2-3, and L3-4, with 1 case each. Another 11 at the L4-5 level and 8 at L5-S1 were surgically treated with the full-endoscopic OLLIF.

All operations were completed as intended. In no patient was intraoperative conversion to another surgical technique necessary. The average operation time recorded in the 20 patients was 131.5 minutes (85-248 minutes), of which, on average, 56.8 minutes were taken up by the directly visualized endoscopic intervertebral body fusion. The average percutaneous pedicle screw system implantation time was 33.6 minutes. Decompression procedure was not needed in 5 surgical segments as no neural compression combined. In 3 segments with central stenosis, the unilateral laminotomy for bilateral decompression decompression took an average time of 58.3 minutes. The average transforaminal decompression time in 14 segments was 33.1 minutes. There were no complications such as nerve injury or dura tear during the operation, infection, cerebrospinal fluid leakage, or failure to cure due to 
Table 1. Changes in the quantitative indices at various time points after full-endoscopic oblique lateral lumbar interbody fusion. (All values are mean \pm standard deviation.)

\begin{tabular}{|c|c|c|c|c|c|c|}
\hline \multirow[b]{2}{*}{ Indices } & \multirow[b]{2}{*}{ Preoperative } & \multicolumn{4}{|c|}{ Postoperative } & \multirow[b]{2}{*}{$\boldsymbol{P}$} \\
\hline & & 1 week & 3 months & 6 months & 1 year & \\
\hline VAS of low back pain & $50.50 \pm 7.76$ & $18.25 \pm 6.54^{\mathrm{a}}$ & $11.50 \pm 6.51^{\mathrm{a}}$ & $5.75 \pm 6.34^{\mathrm{a}}$ & $4.00 \pm 7.54^{\mathrm{ab}}$ & .000 \\
\hline VAS of leg pain & $54.50 \pm 26.99$ & $6.00 \pm 7.36^{\mathrm{a}}$ & $2.00 \pm 4.10^{\mathrm{a}}$ & $3.75 \pm 5.35^{\mathrm{a}}$ & $1.50 \pm 3.66^{\mathrm{a}}$ & .000 \\
\hline ODI & $58.10 \pm 11.82$ & Missing & $22.80 \pm 4.40^{\mathrm{a}}$ & $19.55 \pm 6.36^{\mathrm{a}}$ & $16.55 \pm 4.62^{\mathrm{ab}}$ & .000 \\
\hline
\end{tabular}

Abbreviations: ODI indicates Oswestry disability index; VAS, visual analog scale.

${ }^{a}$ LSD test, $P<.01$, compared with preoperative.

${ }^{b}$ LSD test, $P<.01$, compared with 3 months postoperative.

insufficient decompression, or deteriorating nerve root function. Dysesthesia rising to the level of needing interventional treatment beyond the scope of activity modification, nonsteroidal anti-inflammatories, and physical therapy did not occur in our series. Postoperative lumbar MRI scans routinely performed in all patients confirmed sufficient nerve decompression observed intraoperatively during the directly visualized endoscopic decompression.

All the patients obtained complete follow-up data, which confirmed significant relief of low back pain and leg pain. These VAS score reductions were matched by statistically significant ODI scores improvements $(P<.01$; Table 1$)$. At 1 -year final follow-up, preoperative impaired sensation and muscle strength of the involved nerve root were significantly recovered $(P<.05)$, but tendon reflexes were not significantly improved $(P>.05$; Table 2$)$. At the 1-year follow-up, excellent Macnab outcomes were obtained 13 patients, good in 6 , and fair in 1 . According to the CT-based classification of spinal fusion used by Mannion et al, ${ }^{8}$ CT showed complete interbody fusion in 17 segments and partial fusion in 5 segments at the 6-month follow-up. Complete fusion was achieved in all 22 segments at a 1-year final follow-up.

\section{DISCUSSION}

OLLIF is a lumbar interbody fusion surgery procedure through the Kambin triangle. In open or

Table 2. Changes in nerve root function at 1-year follow-up after fullendoscopic oblique lateral lumbar interbody fusion.

\begin{tabular}{lccc}
\hline Indices & Preoperative & $\mathbf{1 ~ y}$ Postoperative & $\boldsymbol{P}$ \\
\hline Sensation & & & .013 \\
Normal & 11 & 18 & $\ldots$ \\
Abnormal & 9 & 2 & $\ldots$ \\
Muscle strength & & & .037 \\
Normal & 14 & 19 & $\ldots$ \\
Abnormal & 6 & 1 & $\ldots$ \\
Tendon reflex & 13 & 16 & $\ldots$ \\
Normal & 7 & 4 & $\ldots$ \\
Abnormal & & & \\
\hline
\end{tabular}

minimally invasive transforaminal lumbar interbody fusion (TLIF) surgery, the facet joints are usually completely removed to obtain the full exposure of the Kambin triangle. However, the size of the Kambin triangle may be large enough for the insertion of a nonexpandable fusion cage without removing the entire facet. ${ }^{9}$ Thus, preserving some inherent stability that differs in individual patients may be feasible. Patients who underwent the OLLIF operation through the posterolateral transforaminal approach may develop postoperative dysesthesia due to irritation of the dorsal root ganglion of the exiting nerve root. A preventive foraminoplasty was recommended by Katzell $^{2}$ because he observed transient postoperative paresthesias in $20 \%-25 \%$ of his initial cases.

Our clinical series corroborates the utility of the preventive percutaneous foraminoplasty full-endoscopic technique in not only managing the surgical access to the intervertebral disc space but also to protect and visualize the exiting nerve root. Only 2 of the 9 patients had persistent decreased sensory function in the lower extremity at the 1-year followup, neither one of which was caused by the fullendoscopic OLLIF procedure. In comparison, the clinical series of 568 segment OLLIF surgeries published by Abbasi et $\mathrm{al}^{10}$ reported $15(5 \%)$ patients who had residual symptoms of mild nerve root irritation. One patient had significant neurological dysfunction (3/5) at 1-year follow-up and 14 $(4.7 \%)$ patients underwent reoperation within 1 year. Abbasi et $\mathrm{al}^{11}$ also performed OLLIF for 37 patients with degenerative lumbar scoliosis. Cobbs angle decreased from $16^{\circ}$ to $9.3^{\circ}(P<.001)$, equivalent to $2.5^{\circ}$ of OLLIF surgical correction per segment. In that series, there were 3 cases of mild nerve irritation or nerve injury. In comparison, postoperative dysesthesia was not a real issue in our patient series and no patient required any other treatment for it other than supportive care measures during the immediate postoperative recovery period. 
We attributed this observation to the previously described use of a trephine system designed to fully expose the Kambin triangle, and to protect the exiting and the traversing nerve root. ${ }^{4-6}$ As a result, no complications from nerve injury occurred in this study. During the directly visualized endoscopy of the intervertebral disc space, thorough preparation of endplates down to the subchondral bone could be performed to facilitate the intervertebral fusion. This technique may help avoid injury to the endplate common to nonvisualized fusion procedures where decortication into the subchondral bone may contribute to cage subsidence and subsequently to inferior clinical outcomes. ${ }^{12-16}$

Bone grafting materials currently used in fullendoscopic lumbar interbody fusion surgery include autogenous local decompression bone, ${ }^{13,14,17}$ autologous iliac bone, ${ }^{18-20}$ allogeneic cancellous bone, allogeneic decalcified bone matrix, ${ }^{12,19,21,22}$ artificial bone $\beta$-tricalcium phosphate, ${ }^{22,23}$ rhBMP-2, ${ }^{12,21,24}$ etc. Because the harvesting of autologous iliac bone may lead to postoperative pain, infection, and other complications it was avoided in our series, recognizing that newer methods of harvesting iliac bone graft will mitigate autograft harvesting. Therefore, the composite bone grafting method using local decompression autogenous bone combined with other bone grafting materials were chosen, especially using rhBMP-2 to significantly improve the bone graft fusion rate and fusion speed. ${ }^{12,21,24}$

Lumbar interbody fusion under full endoscopy, without the use of an interbody fusion cage, has only reported by a few. ${ }^{20,24}$ Most clinical studies reported using different types of lumbar interbody fusion cages, including a metal expandable fusion cage, ${ }^{18,19,22,25}$ metal non-expandable fusion cage, ${ }^{26}$ PEEK material non-expandable fusion cage, ${ }^{13,14,16,17,27}$ and polymer mesh expandable fusion cage. ${ }^{12,21,28}$ PEEK material, because its elastic modulus is close to that of the lumbar vertebral bone, plays a substantial supporting role between vertebral bodies, and does not affect the judgment of interbody fusion on imaging. It has been the most currently accepted interbody fusion cage material after years of clinical screening of material selections. PEEK nonexpandable fusion cages used for full-endoscopic lumbar interbody fusion have undergone various modifications in shape and size. The head of the fusion cage is often designed as a bullet head. The width of the fusion cage is usually $9 \mathrm{~mm} .{ }^{16,29}$ The metal vertical or bidirectional expandable interbody fusion cage has the characteristics matching the small size of the annular access for insertion through the Kambin triangle into the intervertebral disc space. In its final expanded form it matches the larger footprint best for facilitating interbody fusion. The expansion may be executed to the extent needed to provide sufficient support between the vertebral bodies and the indirect decompression of the spinal canal. ${ }^{18,25}$ A cylindrical expandable interbody fusion cage fits the tubular working sheath of the endoscope, is less likely to occupy the space of the Kambins triangle when entering the intervertebral space, and has higher neurological safety. ${ }^{25}$ PEEK nonexpandable fusion cages and metallic expandable cages have their advantages, and they may play a role in fullendoscopic lumbar interbody fusion surgery in the future. The new generation of bidirectionally expandable cages will have the ability to expand both in height and width to optimize disc space coverage. Although Morgenstern and Morgenstern $^{22}$ reported no significant differences in outcome observed between an expandable and a rigid cage, their pros and cons will require larger samples, prospective controlled studies, and long-term follow-up results to screen these devices better for their suitability for the full-endoscopic lumbar interbody fusion. The OLLIF cage application shown by the authors (Bonovo) is a stepping stone in developing such suitable devices that facilitate the expansion of the clinical indications for the endoscopic spinal surgery from decompression to spinal reconstruction procedures. This conclusion is corroborated by the authors' excellent and good Macnab outcomes in nearly all study patients.

Our fusion rate $(100 \%)$ is similar to those reported in the original studies by Abbasi et al, ${ }^{10,11}$ ranging between $98.7 \%$ and $100 \%$. It compares favorably with other clinical studies of full-endoscopic lumbar interbody fusions, which reported lower fusion rates ranging from $59.6 \%$ to $100 \% .^{12,18,20,21,23-26,28}$ While the fusion rate is related to the quality of the bone graft bed, bone graft material, the choice of interbody fusion cage, the supplemental fixation method, the fusion evaluation method, and the follow-up duration, the use of rh-BMP-2, the impaction of bone allograft anterior in the intervertebral space, and the use of supplemental posterior fixation may have contributed to the high fusion rate in our study. ${ }^{12,20,21,24-28}$ The importance of placing poste- 
rior supplemental pedicle screw constructs to back up nonexpandable fusion to achieve a higher fusion rate has been pointed out by other investigators. ${ }^{19,22,25}$

For patients who need nerve decompression surgery, it is necessary to choose the appropriate decompression method and approach. Full-endoscopic decompression surgery through a transforaminal approach can cover most of the lumbar disc herniation, intervertebral foramen stenosis, and lateral recess stenosis. ${ }^{16,18,21,22,25,30}$ Full-endoscopic lumbar decompression through interlaminar approach is mainly applicable to L5-S1 discectomy and dorsal decompression of severe central spinal stenosis in all lumbar segments. ${ }^{12,13,17,31}$ Safe and effective full-endoscopic lumbar decompression techniques make the contraindications for classic OLLIF into indications for full-endoscopic OLLIF.

Besides, Abbasi and Murphy ${ }^{32}$ retrospectively comparatively analyzed 124 operations (OLLIF = 69 , TLIF $=55$ ). Single-segment surgery time (OLLIF 62.9 minutes versus TLIF 134.9 minutes) and surgical cost (OLLIF \$5,253 versus TLIF $\$ 11,264)$ were reduced in the OLLIF population. Hospital costs (OLLIF \$5,712, TLIF \$9,271) and length of stay also decreased (OLLIF 2.6 days versus TLIF 4.2 days). Reduced resource consumption per patient indicates lower total hospital costs. For hospitals with limited resources, OLLIF surgery can reduce surgical time and length of stay, and can increase the use of each operating room and each bed.

Although our study is clearly limited by the small number of cases, it showed the feasibility of performing successful full-endoscopic OLLIF with a bullet-shaped nonexpandable PEEK cage in conjunction with ample bone grafting and posterior supplemental fixation. However, it was limited by the small number of patients. Our prospective observational study also suffered from short-term follow-up and the absence of a control group. Therefore, the authors recommend to validate its safety and long-term clinical and radiographic outcome in a larger scale comparative study with long-term follow-up and randomization protocols wherever possible.

\section{REFERENCES}

1. Abbasi H, Abbasi A. Oblique lateral lumbar interbody fusion (OLLIF): technical notes and early results of a single surgeon comparative study. Cureus. 2015;7(10):e351. doi:10. 7759 /cureus.351

2. Katzell J. Endoscopic foraminal decompression preceding oblique lateral lumbar interbody fusion to decrease the incidence of post operative dysaesthesia. Int J Spine Surg. 2014:8:1-9. doi:10.14444/1019

3. Yeung AT, Tsou PM. Posterolateral endoscopic excision for lumbar disc herniation: surgical technique, outcome, and complications in 307 consecutive cases. Spine (Phila Pa 1976). 2002;27(7):722-731.

4. Li ZZ, Hou SX, Shang WL, et al. New instrument for percutaneous posterolateral lumbar foraminoplasty: case series of 134 with instrument design, surgical technique and outcomes. Int J Clin Exp Med. 2015;8(9):14672-14679.

5. Li ZZ, Hou SX, Shang WL, et al. Percutaneous lumbar foraminoplasty and percutaneous endoscopic lumbar decompression for lateral recess stenosis through transforaminal approach: technique notes and 2 years follow-up. Clin Neurol Neurosurg. 2016;143:90-94.

6. Li ZZ, Hou SX, Shang WL, et al. Modified percutaneous lumbar foraminoplasty and percutaneous endoscopic lumbar discectomy: instrument design, technique notes, and 5 years follow-up. Pain Physician. 2017;20:E85-E98.

7. Komp M, Hahn P, Merk H, et al. Bilateral operation of lumbar degenerative central spinal stenosis in full-endoscopic interlaminar technique with unilateral approach: prospective 2year results of 74 patients. J Spinal Disord Tech. 2011;24(5):281-287.

8. Mannion RJ, Nowitzke AM, Wood MJ. Promoting fusion in minimally invasive lumbar interbody stabilization with low-dose bone morphogenic protein-2-but what is the cost? Spine J. 2011;11(6):527-533.

9. Hardenbrook M, Lombardo S, Wilson MC, et al. The anatomic rationale for transforaminal endoscopic interbody fusion: a cadaveric analysis. Neurosurg Focus. 2016;40(2):E12. doi:10.3171/2015.10.FOCUS15389

10. Abbasi A, Khaghany K, Orandi V, et al. Clinical and radiological outcomes of oblique lateral lumbar interbody fusion. Cureus. 2019;11(2):e4029. doi:10.7759/CUREUS.4029

11. Abbasi H, Miller L, Abbasi A, et al. Minimally invasive scoliosis surgery with oblique lateral lumbar interbody fusion: single surgeon feasibility study. Cureus. 2017;9(6):e1389. doi:10. 7759/CUREUS.1389

12. Shen J. Fully endoscopic lumbar laminectomy and transforaminal lumbar interbody fusion under local anesthesia with conscious sedation: a case series. World Neurosurg. 2019;127:e745-e750.

13. Heo DH, Park CK. Clinical results of percutaneous biportal endoscopic lumbar interbody fusion with application of enhanced recovery after surgery. Neurosurg Focus. 2019;46(4):E18. doi:10.3171/2019.1.FOCUS18695

14. Kim JE, Choi DJ. Biportal endoscopic transforaminal lumbar interbody fusion with arthroscopy. Clin Orthop Surg. 2018;10(2):248-252.

15. Heo DH, Kim JS. Clinical and radiological outcomes of spinal endoscopic discectomy-assisted oblique lumbar interbody fusion: preliminary results. Neurosurg Focus. 2017;43(2):E13. doi:10.3171/2017.5.FOCUS17196

16. Youn MS, Shin JK, Goh TS, et al. Full endoscopic lumbar interbody fusion (FELIF): technical note. Eur Spine J. 2018;27(8):1949-1955.

17. Park MK, Park SA, Son SK, et al. Clinical and 
radiological outcomes of unilateral biportal endoscopic lumbar interbody fusion (ULIF) compared with conventional posterior lumbar interbody fusion (PLIF): 1-year follow-up. Neurosurg Rev. 2019;42(3):753-761.

18. Yang J, Liu C, Hai Y, et al. Percutaneous endoscopic transforaminal lumbar interbody fusion for the treatment of lumbar spinal stenosis: preliminary report of seven cases with 12-month follow-up. Biomed Res Int. 2019;2019:3091459. doi:10.1155/2019/3091459

19. Lee SH, Erken HY, Bae J. Percutaneous transforaminal endoscopic lumbar interbody fusion: clinical and radiological results of mean 46-month follow-up. Biomed Res Int. 2017;2017:3731983. doi:10.1155/2017/3731983

20. Leu HF, Hauser RK, Schreiber A. Lumbar percutaneous endoscopic interbody fusion. Clin Orthop Relat Res. 1997;337:58-63.

21. Kolcun JPG, Brusko GD, Basil GW, et al. Endoscopic transforaminal lumbar interbody fusion without general anesthesia: operative and clinical outcomes in 100 consecutive patients with a minimum 1-year follow-up. Neurosurg Focus. 2019;46(4):E14. doi:10.3171/2018.12.FOCUS18701

22. Morgenstern R, Morgenstern C. Percutaneous transforaminal lumbar interbody fusion (ptlif) with a posterolateral approach for the treatment of denegerative disk disease: feasibility and preliminary results. Int J Spine Surg. 2015;9:41. doi: $10.14444 / 2041$

23. Nakamura S, Taguchi M. full percutaneous lumbar interbody fusion: technical note. J Neurol Surg A Cent Eur Neurosurg. 2017;78(6):601-606.

24. Osman SG. Endoscopic transforaminal decompression, interbody fusion, and percutaneous pedicle screw implantation of the lumbar spine: a case series report. Int J Spine Surg. 2012;6:157-166.

25. Lewandrowski KU, Ransom NA, Ramirez Leon JF, et al. The concept for a standalone lordotic endoscopic wedge lumbar interbody fusion: the LEW-LIF. Neurospine. 2019;16(1):82-95.

26. Jacquot F, Gastambide D. Percutaneous endoscopic transforaminal lumbar interbody fusion: is it worth it? Int Orthop. 2013;37(8):1507-1510.

27. Heo DH, Son SK, Eum JH, et al. Fully endoscopic lumbar interbody fusion using a percutaneous unilateral biportal endoscopic technique: technical note and preliminary clinical results. Neurosurg Focus. 2017;43(2):E8. doi:10.3171/ 2017.5.FOCUS17146

28. Wang MY, Grossman J. Endoscopic minimally invasive transforaminal interbody fusion without general anesthesia: initial clinical experience with 1-year follow-up. Neurosurg Focus. 2016;40(2):E13. doi:10.3171/2015.11.FOCUS15435

29. Ling Q, He E, Zhang H, et al. A novel narrow surface cage for full endoscopic oblique lateral lumbar interbody fusion: a finite element study. J Orthop Sci. 2019;24(6):991-998.

30. Morgenstern C, Yue JJ, Morgenstern R. Full percutaneous transforaminal lumbar interbody fusion using the facetsparing, trans-Kambin approach. Clin Spine Surg. 2019;33(1):40-45.

31. Kim HS, Paudel B, Jang JS, et al. Percutaneous full endoscopic bilateral lumbar decompression of spinal stenosis through uniportal-contralateral approach: techniques and preliminary results. World Neurosurg. 2017;103:201-209.

32. Abbasi H, Murphy CM. Economic performance of oblique lateral lumbar interbody fusion (OLLIF) with a focus on hospital throughput efficiency. Cureus. 2015;7(7):e292. doi:10.7759/CUREUS.292

Disclosures and COI: There was no external funding in the preparation of this manuscript. Each author certifies that he or she, or a member of his or her immediate family, has no commercial association (ie, consultancies, stock ownership, equity interest, patent/licensing arrangements, etc) that might pose a conflict of interest in connection with the submitted manuscript.

Corresponding Author: Zhen-Zhou Li, MD, Fourth Medical Center of Chinese PLA General Hospital, Department of Orthopedic Surgery. No. 51, Fucheng Rd, Haidian District, Beijing 100048, China. Phone: 86 13601266970; Email: dr_lizhenzhou@163.com.

Published 1 June 2021

This manuscript is generously published free of charge by ISASS, the International Society for the Advancement of Spine Surgery. Copyright (c) 2021 ISASS. To see more or order reprints or permissions, see http://ijssurgery.com. 